\section{ORIGINAL RESEARCH}

L. Saba

R. Sanfilippo

R. Montisci

G. Mallarini

\title{
Assessment of Intracranial Arterial Stenosis with Multidetector Row CT Angiography: A Postprocessing Techniques Comparison
}

\begin{abstract}
BACKGROUND AND PURPOSE: It was demonstrated the some patients with stroke have intracranial stenosis of $50 \%$ or greater and the identification of intracranial arterial stenosis is extremely important in order to plan a correct therapeutical approach. The aim of this study was to assess the image quality and intertechnique agreement of various postprocessing methods in the detection of intracranial arterial stenosis.
\end{abstract}

\begin{abstract}
MATERIAL AND METHODS: Eighty-five patients who were studied by using a multidetector row CT scanner were retrospectively analyzed. A total of 2040 segments were examined in the 85 subjects. Intracranial vasculature was assessed by using MPR, CPR, MIP, and VR techniques. Two radiologists reviewed the CT images independently. Cohen weighted $\kappa$ statistic was applied to calculate interobserver agreement and for image accuracy for each reconstruction method. Sensitivity, specificity, PPV, and NPV were also calculated by using the consensus read as the reference.
\end{abstract}

RESULTS: Two hundred fifteen (10.5\%) stenosed artery segments were identified by the observers in consensus. The best intermethod $\kappa$ values between observers 1 and 2 were obtained by VR and MIP ( $\kappa$ values of 0.878 and 0.861 , respectively), whereas MPR provided the lowest value ( $\kappa$ value of 0.282 ). VR showed a sensitivity for detecting stenosed segments of $88.8 \%$ and $91.6 \%$ for observers 1 and 2 , respectively. The highest positive predictive value was also obtained by VR at $95 \%$ and $99 \%$ for observers 1 and 2, respectively. Image accuracy obtained by using VR was the highest among all reconstruction methods in both observers (185/255 and 177/255 for observers 1 and 2, respectively).

concLusions: The results of our study suggest that VR and MIP techniques provide the best interobserver and intertechnique concordance in the analysis of intravascular cranial stenosis.

\begin{abstract}
ABBREVIATIONS: $\mathrm{CPR}=$ curved planar reconstruction; $\mathrm{CTA}=\mathrm{CT}$ angiography; $\mathrm{DSA}=$ digital subtraction angiography; $\mathrm{HU}=$ Hounsfield unit; ICA = internal carotid artery; MDCTA = multidetector row CT angiography; MIP = maximum intensity projection; MPR = multiplanar reconstruction; MRA = MR angiography; NASCET = North American Symptomatic Carotid Endarterectomy Trial; $\mathrm{NC}=$ not calculated; NPV = negative predictive value; $\mathrm{PPV}=$ positive predictive value; $\mathrm{VR}=$ volume rendering
\end{abstract}

S troke is the third leading cause of mortality and the leading cause of morbidity in the United States and it is usually determined by extracranial carotid pathology. ${ }^{1-3}$ In fact, it has been demonstrated that some patients with stroke show intracranial stenosis of $50 \%$ or greater ${ }^{4}$ and that the identification and characterization of intracranial arterial stenosis is extremely important to plan the correct therapeutic approach.

DSA has long been considered the criterion standard for imaging evaluation of intracranial stenosis and occlusion even if MDCTA and MRA have been increasingly used as feasible noninvasive techniques to evaluate the intracranial vasculature. In particular, the ongoing development of MDCTA technology has transformed CT into a 3D imaging technique with outstanding temporal and spatial resolution and the capacity for visualization of even millimeter-sized vessels such as the Adamkiewicz artery. ${ }^{5,6}$

By using MDCT, it is possible to employ different postpro-

Received May 29, 2009; accepted after revision September 3

From the Department of Radiology (L.S., G.M.) and Department of Vascular Surgery (R.S., R.M., G.M.), Policlinico Universitario, University of Cagliari, Monserrato (Cagliari), Italy.

Please address correspondence to Luca Saba, Department of Radiology Policlinico Universitario, University of Cagliari s.s. 554 Monserrato (Cagliari), 09045 Italy; e-mail: lucasaba@ tiscali.it

DOI 10.3174/ajnr.A1976 cessing techniques in addition to source axial images. MIP, $\mathrm{MPR}, \mathrm{CPR}$, and $\mathrm{VR}^{7,8}$ are currently the most frequently used. A deeper knowledge of the potential of postprocessing techniques and their application will allow optimization of the MDCTA dataset. To the best of our knowledge no previous studies have compared these tools for the assessement of intracranial vasculature.

The purpose of this study was to assess the image quality and intertechnique agreement of different MDCTA postprocessing techniques in the analysis of intracranial arterial stenosis.

\section{Materials and Methods}

\section{Patient Population}

The study group was selected following a search in the patient database of the Azienda Ospedaliero-Universitaria di Cagliari, Polo di Monserrato (previously Policlinico Universitario di Monserrato). Study patients were enrolled from those subjects who had undergone a previous CTA for the study of supra-aortic vessel and intracranial vasculature from January 2007 to June 2008. The study cohort included 85 patients ( 67 men, 18 women; mean age, 68 years; age range, $49-86$ years) who had been studied by using a multidetector row CT scanner.

This is a retrospective study, and each MDCTA examination was 

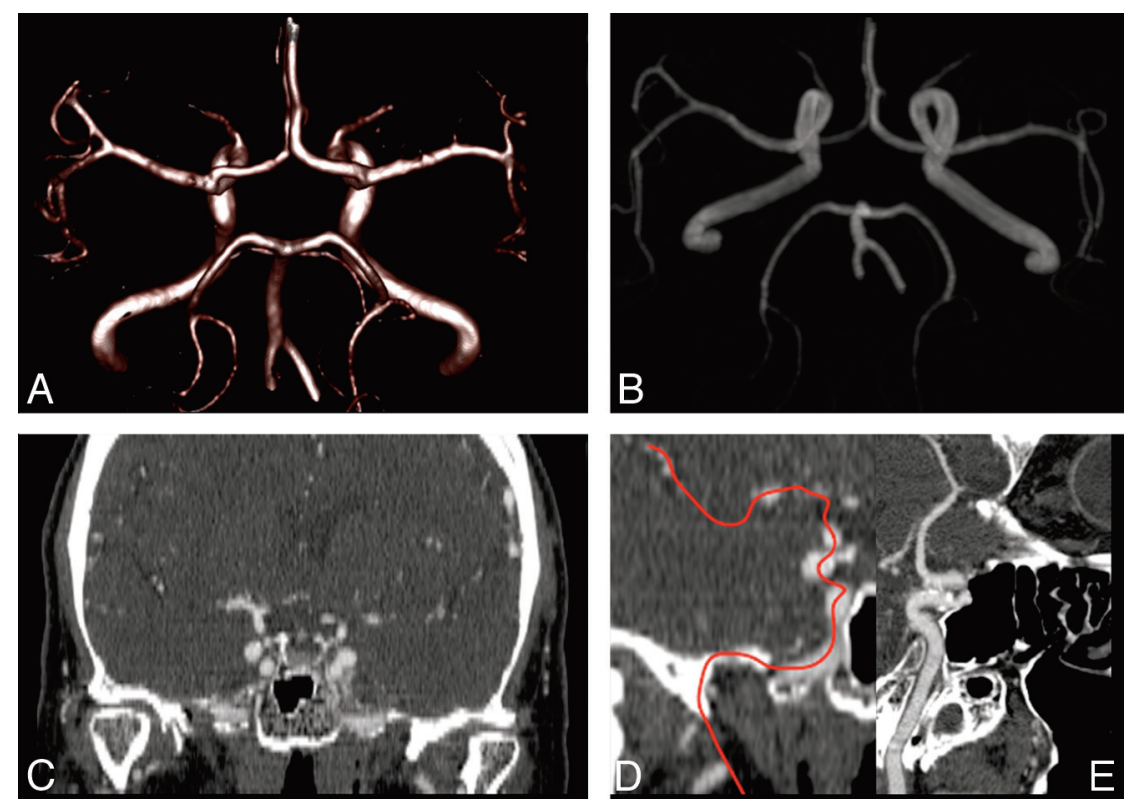

Fig 1. Sixty-three-year-old female patient: postprocessed VR $(A), \operatorname{MIP}(B)$, corona MPR $(C)$, and CPR images $(D, E)$.

performed when it was clinically indicated, and it was ordered by the patient's physician as part of routine clinical care. In our institute, the inclusion criterion for performing MDCTA is as follows: a prior given clinical indication for CT angiography of the supra-aortic vessels (when possible also confirmed by ultrasound echo color Doppler study) as stated by the referring physician and established by the attending radiologist. In particular, the main reason for referral to MDCTA is a sonography (ultrasound) examination that showed a pathologic stenosis and/or a plaque alteration or when ultrasound cannot provide sufficient information about the degree of stenosis, as for example in the presence of large calcified plaques with acoustic shadowing, high carotid bifurcation, or hostile neck (edema, obese patients). Plaque alteration was considered as the presence of a heterogeneous plaque, an irregular surface, intraplaque hemorrhage, and/or the presence of ulceration in the plaque. Carotid arteries of asymptomatic patients were studied in our department in diabetics $>50$ years old and in patients who underwent cardiac interventions for coronary artery disease, aortic interventions, and lower leg artery surgery.

Exclusion criteria for the study consisted of contraindications to iodinated contrast media, such as a known allergy to iodinated contrast material, or elevated renal function tests.

Because this study was retrospective and the imaging undertaken was not additional to that performed routinely in this group of patients, it is the policy of our divisional research committee that specific ethical approval was not required for this study. Some of our patients had also been included in previous studies blinded for peer review purposes.

\section{MDCTA Study of Carotid Arteries}

Examinations were performed by using a 4-detector row CT scanner (Philips MX8000; formerly Picker, Andover, Massachusetts). After receiving information about the type of investigation, each patient was asked to sign an informed consent for contrast administration. Patients were placed in the supine position, with the head tilted back to prevent dental artifacts on the images and with the arms along the chest. Patients were also instructed not to breathe and not to swallow.
A scout view including thorax, neck, and skull was acquired. The CTA scan range encompassed the ascending aorta to the intracranial blood vessels ( $5 \mathrm{~cm}$ above the sella turcica) because in our center head and neck CTAs are routinely combined. Arterial enhancement was provided by the intravenous administration of $90-140 \mathrm{~mL}$ of contrast material (Ultravist 370; Schering, Berlin, Germany) at an injection speed of $4-6 \mathrm{~mL} / \mathrm{s}$ by using a power injector and an 18 - to 20 -gauge intravenous catheter inserted into the antecubital vein.

A delay time variable from 12 to 18 seconds was applied.

Images were obtained by using a helical acquisition in caudocranial scan direction with a section thickness of $1.3 \mathrm{~mm}$, an increment of $0.6 \mathrm{~mm}$, a matrix of $512 \times 512$, and a FOV of $16-19 \mathrm{~cm}(260-300$ mAs; $120-140 \mathrm{kV})$.

\section{Postprocessing Methods}

Images were processed with our workstations (Philips Dell Precision 690, Intel Xeon processor) by using MPR, MIP, CPR, and VR algorithms (Fig 1). MPR is probably the most simple and commonly applied reformatting method and consists of obtaining the average value of the attenuation of the pixels along the axis of projection. The vascular MPR image is 2D, and for this reason it is not possible to analyze the vessel course. ${ }^{9}$ Multiple projections for each dataset were freely generated, and radiologists could manipulate the window, the center, and the view angle.

CPR depicts the cross-sectional profile of a vessel along its length while preserving the relative $\mathrm{x}$-ray attenuation. Through a series of contiguous source images on each image the course of the target vessel is traced by a series of mouse clicks. ${ }^{10}$ This procedure continues until the entire course of the vessel has been traced. CPR is time consuming, and small deviations from the true centerline, particularly in narrow arteries, can generate false stenosis or, on the contrary, can cause important lesions to be missed. ${ }^{11}$

VR is one of the most advanced and computer-intensive rendering algorithms available, incorporating all relevant data into the resulting image, and it produces high-quality $3 \mathrm{D}$ angiographic images. VR images were generated by selecting trapezoids relative to the voxel intensity histogram, ${ }^{12}$ and then an opacity value was assigned to de- 
fine its relative transparency. To remove bones we used 2 distinct procedures: the automatic bone removal function, followed by the manual cutting function. The automatic bone removal function was set to exclude only the voxel closest to the selected target to avoid the possibility that vascular structures would be cut off; to complete the area of nonautomatic bone removal, we manually deleted the bones by using the dedicated software.

The dataset with the bone removal was then saved for use in the MIP analysis to have MIPs without superimposition of bones.

MIP is a simple $3 \mathrm{D}$ visualization tool that can be used to display a CTA dataset. MIP images are not threshold dependent and they preserve the whole CT dataset. For a given viewing direction, parallel rays are cast through a volume of interest, and the maximum CT number encountered along each ray is displayed. For this reason, if bone or calcifications are found within the projection ray these structures are represented on the MIP image instead of the contrast-enhanced ves$\operatorname{sel}^{9}$ because of higher attenuation values, and this probably represents the greatest disadvantage of this technique.

Readers could use MIP thick slabs and the thickness was freely modifiable. Moreover, the CT VR bone exclusion obtained from VR analysis was used to generate MIP images without the voxel of the bone structures that had already been removed.

\section{Image Analysis}

The MDCTA images were reviewed for the presence, location, and severity of stenosis by 2 independent observers (observer 1, a radiologist with 5 years of experience in vascular study; observer 2 , a radiologist with $>10$ years of experience in vascular study). Cases were presented in a random order, and both observers were blinded to the patient history and to all clinical information. To prevent recall bias risk, different postprocessed images from the same patient were presented to the readers separately; first, all MPR postprocessed images were analyzed, followed by VRs, CPRs, and MIPs, respectively. While evaluating the individual postprocessed dataset, readers did not have access to the source images.

In the evaluation of all imaging modalities, the NASCET criteria were used for stenosis calculations, as previously reported ${ }^{13}:\left[\left(D_{\mathrm{n}}-\right.\right.$ $\left.\left.D_{\mathrm{s}}\right) / D_{\mathrm{n}}\right] \times 100$, where $D_{\mathrm{n}}$ is normal diameter and $D_{\mathrm{s}}$ is stenosed diameter. We considered the vessel distal to the stenosis as normal for stenosis.

NASCET stenoses were grouped according to the following grading scale ${ }^{9}$ : normal $(0 \%-9 \%)$, mild $(10 \%-29 \%)$, moderate $(30 \%-$ $69 \%)$, severe (70\%-99\%), or occluded, and diseased vessels were defined as those whose NASCET stenosis rates fell into the moderate, severe, or occluded categories. ${ }^{13}$

The following bilateral arterial segments were included ${ }^{13}$ : high cervical ICA, petrous ICA, cavernous ICA, supraclinoid ICA, A1, A2, M1, M2, intracranial vertebral, P1, and P2, as well as the proximal basilar and distal basilar arteries. Therefore, a total of 2040 vessel segments were examined in the 85 subjects. When an intracranial artery was uniformly and segmentally of decreased caliber throughout its length when compared with the contralateral side, this vessel segment was considered hypoplastic and no stenosis was attributed. ${ }^{13}$

After the independent reading of the CTA dataset, observers reanalyzed data in consensus by using axial CTA images and postprocessing procedures to develop a reference standard. Both of the observers discussed each CTA dataset 1 month after their independent analysis and in the case of discrepant opinion, new measurements were reobtained by considering the axial CTA images and the postprocessing procedures.
The observers classified the quality of the 4 postprocessed datasets in each patient by grouping them into 4 categories: poor, suboptimal for diagnostic purposes (0); fair, only adequate for diagnostic purposes (1); good but slightly lower quality compared with excellent, but useful for diagnostic purposes (2); and excellent, high quality for diagnostic purposes (3).

In the final phase we measured the HU value in each ICA and we correlated this value with the quality image. To calculate the HU value each reader measured the HU values in the high cervical ICA by using a circular or elliptical region-of-interest cursor. HU opacification was then classified according to 3 categories: $>400 \mathrm{HU},>250$ to $<400$ $\mathrm{HU}$, and $<250 \mathrm{HU}$.

\section{Statistical Analysis}

The Kolmogorov-Smirnov $Z$-test was used to test the normality of continuous variable groups. Interobserver agreement and the weighted $\kappa$ value between observers in the evaluation of MIP, MPR, $\mathrm{CPR}$, and VR image quality were assessed.

Interobserver agreement analysis was performed by calculating the observed agreement (percentage of the observations) and by calculating the weighted $\kappa$ index value with its $95 \%$ confidence intervals. A $\kappa$ value of $0-0.20$ indicated poor agreement, $0.21-0.40$ indicated fair agreement, $0.41-0.60$ indicated moderate agreement, $0.61-0.80$ showed good agreement, and 0.81-1.00 meant very good agreement.

To assess interobserver reliability in the stenosis quantification, the calculated stenosis measurements of the first reviewer were compared with those of the second reviewer for each reconstruction tool and the $\kappa$ value was then calculated. To produce these data, R software (www.r-project.org) was employed. A $P$ value of $<.05$ was regarded as indicating statistical significance, and all correlation values were calculated by using a 2-tailed significance level.

\section{Results}

\section{General Results}

We analyzed 2040 intracranial arterial segments, and 215 (10.5\%) stenosed artery segments were identified by the observers in consensus. Ninety-two stenoses were identified in anterior circulation vessels, whereas 123 stenoses were found in posterior circulation vessels; the distribution of stenosed artery segments is summarized in Table 1. Only 6 stenoses $(2.8 \%)$ were classified as mild, whereas 162 were moderate (75.3\%), 29 were severe (13.5\%), and 18 segments were occluded $(8.4 \%)$. The number of stenosed segments in each patient ranged from 1 to 8 , with a mean of 2.5.

\section{Concordance in the Identification of Stenosed Intracranial Arterial Segments}

Results obtained from the analysis of the postprocessing methods for the detection of stenosed intracranial arterial segments are reported in Tables 2 and 3. Observers 1 and 2 detected the highest number of intracranial arterial segments by using VR (201 and 197, respectively), whereas the lowest total scores were obtained by using MPR ( 85 and 88 for observers 1 and 2, respectively). The best intermethod $\kappa$ values between observers 1 and 2 were obtained by VR and MIP ( $\kappa$ values of 0.878 and 0.861 , respectively), whereas MPR gave the poorest results ( $\kappa$ value of 0.282 ). 


\begin{tabular}{lcc}
\hline $\begin{array}{l}\text { Table 1: Location of intracranial arterial stenosed segments } \\
\text { detected by observers in consensus }\end{array}$ \\
\hline & $\begin{array}{c}\text { No. (\%) of Lesions } \\
(n=215)\end{array}$ & $\begin{array}{c}\text { No. (\%) of Lesions } \\
(n=215)\end{array}$ \\
\hline High cervical ICA right & $7(3.2)$ & $54(25)$ \\
High cervical ICA left & $7(3.2)$ & \\
Petrous ICA right & $9(4.2)$ & \\
Petrous ICA left & $12(6.7)$ & \\
Cavernous ICA right & $6(2.8)$ & \\
Cavernous ICA left & $4(1.9)$ & \\
Supraclinoid ICA right & $4(1.9)$ & \\
Supraclinoid ICA left & $5(2.3)$ & \\
A1 right & $2(0.9)$ & \\
A1 left & $1(0.5)$ & \\
A2 right & $2(0.9)$ & \\
A2 left & $2(0.9)$ & \\
M1 right & $9(4.2)$ & \\
M1 left & $7(3.2)$ & \\
M2 right & $8(3.7)$ & \\
M2 left & $7(3.2)$ & \\
Intracranial vertebral right & $23(10.7)$ & \\
Intracranial vertebral left & $26(12)$ & \\
P1 right & $12(6.7)$ & $47(22)$ \\
P1 left & $8(3.7)$ & \\
P2 right & $11(5.1)$ & \\
P2 left & $16(7.4)$ & \\
Proximal basilar & $13(6)$ & \\
Distal basilar & $14(6.5)$ & \\
\hline
\end{tabular}

Table 2: Number of intracranial arterial stenosed segments according to observers and postprocessing techniques

\begin{tabular}{|c|c|c|c|c|c|c|c|c|}
\hline & \multicolumn{8}{|c|}{ Number of Identified Lesions } \\
\hline & \multicolumn{4}{|c|}{ Observer 1} & \multicolumn{4}{|c|}{ Observer 2} \\
\hline & MPR & CPR & MIP & VR & MPR & CPR & MIP & VR \\
\hline High cervical ICA right & 3 & 5 & 6 & 6 & 3 & 4 & 7 & 7 \\
\hline High cervical ICA left & 3 & 4 & 7 & 7 & 4 & 5 & 7 & 7 \\
\hline Petrous ICA right & 4 & 5 & 5 & 8 & 3 & 4 & 6 & 9 \\
\hline Petrous ICA left & 5 & 6 & 7 & 11 & 2 & 5 & 8 & 11 \\
\hline Cavernous ICA right & 2 & 8 & 5 & 5 & 4 & 4 & 4 & 4 \\
\hline Cavernous ICA left & 3 & 2 & 4 & 3 & 4 & 3 & 4 & 5 \\
\hline Supraclinoid ICA right & 3 & 3 & 4 & 4 & 4 & 2 & 4 & 5 \\
\hline Supraclinoid ICA left & 3 & 4 & 5 & 5 & 2 & 3 & 5 & 4 \\
\hline A1 right & 0 & 1 & 2 & 2 & 0 & 1 & 2 & 3 \\
\hline A1 left & 0 & 0 & 1 & 1 & 1 & 0 & 2 & 1 \\
\hline A2 right & 0 & 1 & 1 & 2 & 0 & 1 & 1 & 2 \\
\hline A2 left & 0 & 0 & 1 & 2 & 0 & 0 & 2 & 2 \\
\hline M1 right & 3 & 6 & 7 & 8 & 2 & 3 & 5 & 6 \\
\hline M1 left & 2 & 4 & 6 & 7 & 3 & 2 & 4 & 7 \\
\hline M2 right & 3 & 4 & 9 & 6 & 3 & 3 & 8 & 8 \\
\hline M2 left & 2 & 1 & 10 & 6 & 2 & 1 & 11 & 7 \\
\hline Intracranial vertebral right & 8 & 15 & 16 & 21 & 6 & 12 & 17 & 21 \\
\hline Intracranial vertebral left & 12 & 14 & 21 & 24 & 9 & 19 & 22 & 25 \\
\hline P1 right & 2 & 5 & 8 & 11 & 3 & 7 & 9 & 12 \\
\hline P1 left & 3 & 6 & 6 & 9 & 4 & 8 & 7 & 7 \\
\hline $\mathrm{P} 2$ right & 5 & 8 & 9 & 14 & 3 & 4 & 8 & 9 \\
\hline P2 left & 6 & 12 & 14 & 13 & 8 & 14 & 13 & 13 \\
\hline Proximal basilar & 7 & 9 & 12 & 14 & 9 & 8 & 10 & 10 \\
\hline Distal basilar & 6 & 9 & 11 & 12 & 9 & 10 & 10 & 12 \\
\hline Total & 85 & 132 & 177 & 201 & 88 & 123 & 176 & 197 \\
\hline
\end{tabular}

\section{Sensitivity, Specificity, PPV, and NPV}

The sensitivity, specificity, PPV, and NPV for observers 1 and 2 are given in Tables 4 and 5. These calculations were based on the consensus reference of both observers who used axial CTA images and postprocessing procedures.
The sensitivity of VR in detecting stenosed segments was $88.8 \%$ and $91.6 \%$ for observers 1 and 2 , respectively. The highest positive predictive value was also obtained by VR at $95 \%$ and $99 \%$ for observers 1 and 2, respectively.

\section{Image Quality Analysis and HU Assessment}

Image quality analysis is summarized in Tables $6-8$. The total image accuracy reached by using VR was the highest among all reconstruction methods in both observers (185/255 and 177/ 255 for observers 1 and 2, respectively), whereas the highest $\kappa$ value was observed by using the MIP ( $\kappa$ value of 0.783 ).

The relationship between the HU value in the intracranial vascular segments and image quality was also studied. We observed a significant statistical correlation between the $\mathrm{HU}$ value $>400$ and optimal image accuracy for both readers $(P=$ 0.0011 and 0.0018 for readers 1 and 2, respectively).

\section{Discussion}

In recent years, DSA has been considered the criterion standard for the examination of the intracranial vasculature, ${ }^{14,15}$ but it has gradually been replaced by noninvasive techniques such as CTA and MRA because of the complication risks. ${ }^{16}$ It has been stated that MDCTA will become the new criterion standard for vascular imaging. ${ }^{17-20}$

Our aim was to assess the image accuracy and intertechnique agreement of 4 postprocessing methods in the detection of intracranial arterial stenosis. In this study 215 (10.5\%) stenosed artery segments were identified and the best intermethod $\kappa$ values between the observers were obtained by VR and MIP, whereas MPR gave the lowest results. VR showed the highest sensitivity in detecting stenosed segments in both observers.

The percentage of stenosed segments identified by the observers was lower than that in the study by Bash and colleagues, ${ }^{13}$ where the percentage of stenosed segments was $17 \%$ (115/672), but was higher than that observed by Skutta and colleagues, ${ }^{20}$ where the percentage of stenosed segments was $4.8 \%(105 / 2205)$. This may be due to the different criterion standard used. Skutta and colleagues employed conventional angiography, whereas we used a multidetector row CT. The selection of the patient population was also different. Additionally, DSA depicts arteries below the detection threshold of MDCTA; the smallest arterial intracranial diameter reliably depicted with MDCTA is $0.7 \mathrm{~mm}$, whereas for DSA it is 0.4 $\mathrm{mm}^{21}$

The percentage of stenosed segments detected in the anterior and posterior circulation was similar in our work and in the study reported by Bash and colleagues. ${ }^{13}$ In the anterior circulation we observed the highest number of stenosed segments in the petrous ICA (10.9\% of stenosed segments), whereas in the posterior circulation the most frequently affected vessels were vertebral arteries $(22.7 \%$ of stenosed segments). The lowest number of stenosed segments was identified in the anterior cerebral arteries (3\%).

The number of stenosed segments in each patient ranged from 1 to 8 , with a mean of 2.5 , whereas Bash and colleagues ${ }^{13}$ reported a variable number of diseased vessel segments with a mean of 4 , a mode of 3 , and a range of 1-13 per patient.

When we analyzed the postprocessing methods for the detection of stenosed intracranial arterial segments we obtained 


\begin{tabular}{|c|c|c|c|c|c|}
\hline & \multicolumn{4}{|c|}{ Observer 1} & \multirow[b]{2}{*}{ Reference } \\
\hline & MIP & MPR & VR & CPR & \\
\hline \multicolumn{6}{|l|}{ Observer 2} \\
\hline MIP & $0.861(0.019)$ & $0.225(0.037)$ & $0.53(0.033)$ & $0.39(0.037)$ & $0.59(0.031)$ \\
\hline MPR & $0.414(0.038)$ & $0.282(0.047)$ & $0.481(0.037)$ & $0.348(0.043)$ & $0.511(0.035)$ \\
\hline VR & $0.627(0.030)$ & $0.36(0.037)$ & $0.878(0.019)$ & $0.543(0.034)$ & $0.951(0.011)$ \\
\hline CPR & $0.414(0.037)$ & $0.282(0.043)$ & $0.566(0.034)$ & $0.611(0.037)$ & $0.635(0.031)$ \\
\hline Reference & $0.653(0.029)$ & $0.397(0.036)$ & $0.909(0.015)$ & $0.552(0.033)$ & NC \\
\hline
\end{tabular}

Note:-Numbers in parentheses indicate SD.

\begin{tabular}{|c|c|c|c|c|}
\hline & Sensitivity & Specificity & PPV & NPV \\
\hline MPR & $0.302(95 \% \mathrm{Cl}=0.241-0.364)$ & $0.989(95 \% \mathrm{Cl}=0.984-0.994)$ & $0.765(95 \% \mathrm{Cl}=0.675-0.885)$ & $0.923(95 \% \mathrm{Cl}=0.911-0.935)$ \\
\hline CPR & $0.474(95 \% \mathrm{Cl}=0.408-0.541)$ & $0.984(95 \% \mathrm{Cl}=0.978-0.989)$ & $0.773(95 \% \mathrm{Cl}=0.701-0.844)$ & $0.941(95 \% \mathrm{Cl}=0.930-0.951)$ \\
\hline MIP & $0.637(95 \% \mathrm{Cl}=0.573-0.701)$ & $0.974(95 \% \mathrm{Cl}=0.967-0.982)$ & $0.745(95 \% \mathrm{Cl}=0.682-0.808)$ & $0.958(95 \% \mathrm{Cl}=0.949-0.967)$ \\
\hline VR & $0.888(95 \% \mathrm{Cl}=0.846-0.930)$ & $0.995(95 \% \mathrm{Cl}=0.991-0.998)$ & $0.950(95 \% \mathrm{Cl}=0.920-0.980)$ & $0.987(95 \% \mathrm{Cl}=0.982-0.992)$ \\
\hline \multicolumn{5}{|c|}{ Table 5: Observer 2: Sensitivity, specificity, NPV, and PPV of the different reconstruction methods } \\
\hline & Sensitivity & Specificity & PPV & NPV \\
\hline MPR & $0.381(95 \% \mathrm{Cl}=0.316-0.446)$ & $0.997(95 \% \mathrm{Cl}=0.994-0.999)$ & $0.824(95 \% \mathrm{Cl}=0.734-0.894)$ & $0.932(95 \% \mathrm{Cl}=0.921-0.943)$ \\
\hline CPR & $0.521(95 \% \mathrm{Cl}=0.454-0.588)$ & $0.994(95 \% \mathrm{Cl}=0.990-0.998)$ & $0.762(95 \% \mathrm{Cl}=0.670-0.884)$ & $0.946(95 \% \mathrm{Cl}=0.936-0.956)$ \\
\hline MIP & $0.572(95 \% \mathrm{Cl}=0.506-0.638)$ & $0.971(95 \% \mathrm{Cl}=0.963-0.979)$ & $0.699(95 \% \mathrm{Cl}=0.631-0.767)$ & $0.951(95 \% \mathrm{Cl}=0.941-0.960)$ \\
\hline VR & $0.916(95 \% \mathrm{Cl}=0.879-0.953)$ & $0.999(95 \% \mathrm{Cl}=0.998-1)$ & $0.995(95 \% \mathrm{Cl}=0.985-1)$ & $0.990(95 \% \mathrm{Cl}=0.986-0.995)$ \\
\hline
\end{tabular}

\begin{tabular}{|c|c|c|c|c|c|}
\hline & \multicolumn{4}{|c|}{ Image Quality } & \multirow[b]{2}{*}{ Total Score } \\
\hline & Poor & Fair & Good & Excellent & \\
\hline MPR & 7 & 29 & 38 & 11 & 138 \\
\hline CPR & 1 & 17 & 44 & 23 & 174 \\
\hline MIP & 6 & 23 & 38 & 18 & 153 \\
\hline VR & 2 & 7 & 50 & 26 & 185 \\
\hline
\end{tabular}

Note:-Poor $=0$, fair $=1$, good $=2$, and excellent $=3$.

\begin{tabular}{|c|c|c|c|c|c|}
\hline & \multicolumn{4}{|c|}{ Image Quality } & \multirow[b]{2}{*}{ Total Score } \\
\hline & Poor & Fair & Good & Excellent & \\
\hline MPR & 5 & 33 & 37 & 10 & 137 \\
\hline CPR & 1 & 15 & 48 & 21 & 174 \\
\hline MIP & 3 & 24 & 46 & 12 & 152 \\
\hline VR & 0 & 13 & 52 & 20 & 177 \\
\hline
\end{tabular}

Note:-Poor $=0$, fair $=1$, good $=2$, and excellent $=3$.

the results that are summarized in Tables 2 and 3. Observers 1 and 2 detected the highest number of intracranial arterial segments by using VR (201 and 197, respectively), and the lowest total scores were obtained by using MPR ( 85 and 88 for observers 1 and 2, respectively). The best intermethod $\kappa$ values between observers 1 and 2 were obtained by VR and MIP ( $\kappa$ value of 0.878 and 0.861 , respectively), whereas MPR gave the poorest results ( $\kappa$ value of 0.282 ).

It is important to mention that several conditions can cause limitations in the identification and grading of stenosis and that these affect each reconstruction method in different ways.

MIP suffers limitations in delineating the lumen of the artery, especially with circumferential wall calcification: in fact, calcification is the limiting factor on MIP images because of

\begin{tabular}{|c|c|c|c|c|}
\hline & \multicolumn{4}{|c|}{ Observer 1} \\
\hline & MIP & MPR & VR & CPR \\
\hline \multicolumn{5}{|c|}{ Observer 2} \\
\hline MIP & $0.783(0.085)$ & $0.596(0.073)$ & $0.406(0.078)$ & $0.416(0.82)$ \\
\hline MPR & $0.544(0.074)$ & $0.676(0.070)$ & $0.407(0.064)$ & $0.239(0.79)$ \\
\hline VR & $0.465(0.076)$ & $0.254(0.074)$ & $0.704(0.070)$ & $0.363(0.083)$ \\
\hline CPR & $0.418(0.79)$ & $0.245(0.079)$ & 0.409 (0.082) & $0.728(0.067)$ \\
\hline
\end{tabular}

the inability of this tool to separate mural calcifications and intramural contrast material.

In some calcified arteries it is very difficult to distinguish occluded segments from stenosed segments and, as a consequence, the exact degree of stenosis. The poor results of MPR were unexpected because this postprocessing procedure usually shows good results in vessel analysis, ${ }^{9,22,23}$ particularly in the presence of calcifications. We hypothesized that the resulting data could be due to the highly tortuous course of the studied vessels. $^{23}$

MPR, CPR, and in particular VR allow adequate analysis of artery vessels with a calcified plaque. Skutta and colleagues ${ }^{20}$ observed that MDCTA showed limitations in grading stenoocclusive lesions in the petrous segments of the carotid artery.

The VR method detected a higher number of stenosed segments compared with MPR, MIP, and CPR (Table 2); for this reason, we assumed that VR would be the best method for the study of the petrous segment of the ICA. We also think that the difference we observed regarding petrous ICA stenosis evaluation compared with those of Skutta and colleagues ${ }^{20}$ may be due to the fact that the surrounding bone was diligently removed in the present study, as described in the Materials and Methods section. Additionally, we employed a 4-detector row CT rather than a helical CT scanner. 
Sensitivity, specificity, PPV, and NPV were assessed by using the consensus analysis of both observers of the axial CTA images, postprocessing procedures, and clinical charts as the criterion standard. VR showed a sensitivity in detecting stenosed segments of $88.8 \%$ and $91.6 \%$ for observers 1 and 2, respectively.

The highest PPV was obtained by VR at 95\% and 99\% for observers 1 and 2, respectively. The results of this study confirm and improve upon previous data reported by other authors that indicate that VR is an advanced visualization method for the assessment of the vascular system. ${ }^{24-26}$

Image accuracy analysis is reported in Tables 6-8. The total image accuracy obtained by using VR was the highest among all reconstruction methods in both observers (185/255 and 177/255 for observers 1 and 2, respectively), whereas the highest $\kappa$ value was observed in MIP ( $\kappa$ value of 0.783 ).

We also observed that an important parameter for producing high-quality images of intracranial vasculature was their HU value; in fact, for both readers, we recorded a significant statistical correlation between an $\mathrm{HU}$ value of $>400$ and optimal image accuracy $(P=0.0011$ and 0.0018 for readers 1 and 2 , respectively). These data also demonstrate the importance of employing all technical methods to obtain high opacification with the contrast material, such as the bolus technique and high concentration of noniodinated contrast material. $^{27,28}$

A deeper knowledge of the potential of postprocessing techniques and their application allows optimization of the MDCTA dataset by reducing wasted time, and our results indicate that VR and MIP are the optimal tools for analyzing intracranial vasculature. To the best of our knowledge there is no reference in the literature of previous studies comparing postprocessing tools for the assessment of intracranial vasculature.

We are aware that our study has some limitations. First, it was a retrospective study and this fact may introduce bias in the data homogeneity. Second, the study was performed by using a 4-detector row CT scanner; however, it has been demonstrated that there are no significant differences in detection rates between 4 -MDCT and $16-\mathrm{MDCT}^{21}$ in the analysis of small intracranial arteries. Third, we did not have a DSA criterion standard; to calculate the diagnostic accuracy, we used the consensus of axial CTA images and postprocessing procedures, and the absence of an accepted criterion standard should be considered in the evaluation of our results.

\section{Conclusions}

On the basis of our results, VR and MIP are the most effective postprocessing techniques and should be recommended for the diagnosis and quantification of intracranial arterial stenosis. A high intraluminal $\mathrm{HU}$ value $(>400 \mathrm{HU})$ should be pursued to achieve optimal image quality.

\section{References}

1. Gibbs RGJ, Todd JC, Irvine C, et al. Relationship between the regional and national incidence of transient ischaemic attack and stroke and performance of carotid endarterectomy. Eur J Vasc Surg 1998;16:47-52
2. Murray CJ, Lopez AD. Morality by cause for eight regions of the world: global burden of disease study. Lancet 1997;349:1269-76

3. Goessens BMB, Visseren FLJ, Kappelle LJ, et al. Asymptomatic carotid artery stenosis and the risk of new vascular events in patients with manifest arterial disease. Stroke 2007;38:1470-75

4. Lutsep HL, Clark WM. Association of intracranial stenosis with cortical symptoms or signs. Neurology 2000;55:716-18

5. Boll DT, Lewin JS, Fleiter TR, et al. Multidetector CT angiography of arterial inflow and runoff in the lower extremities: a challenge in data acquisition and evaluation. J Endovasc Ther 2004;11:144-51

6. Boll TD, Bulow $\mathrm{H}$, Blackman KA, et al. MDCT angiography of the spinal vasculature and the artery of Adamkiewicz. AJR Am J Roentgenol 2006; 187:1054-60

7. Marcus CD, Ladam-Marcus VJ, Bigot JL, et al. Carotid artery stenosis: evaluation at CT angiography with the volume rendering technique. Radiology 1999;211:775-80

8. Corti R, Alerci M, Wyttenbach R, et al. Usefulness of multiplanar reconstructions in evaluation of carotid CT angiography. Radiology 2003;226:290-91

9. Lell MM, Anders K, Uder M, et al. New techniques in CT angiography. Radiographics 2006;26:S45-S62

10. Ochi T, Shimizu K, Yasuhara Y, et al. Curved planar reformatted CT angiography: usefulness for the evaluation of aneurysms at the carotid siphon. AJNR Am J Neuroradiol 1999;20:1025-30

11. Raman R, Napel S, Beaulieau CF, et al. Automated generation of curved planar reformations from volume data: method and evaluation. Radiology 2002;223:275-80

12. Leclerc $X$, Godefroy $\mathrm{O}$, Lucas $\mathrm{C}$, et al. Internal carotid arterial stenosis: CT angiography with volume rendering. Radiology 1999;210:673-82

13. Bash S, Villablanca JP, Jahan R, et al. Intracranial vascular stenosis and occlusive disease: evaluation with CT angiography, MR angiography, and digital subtraction angiography. AJNR Am J Neuroradiol 2005;26:1012-21

14. Chimowitz MI, Kokkinos J, Strong J, et al. The warfarin-aspirin symptomatic intracranial disease study. Neurology 1995;45:1488-93

15. Katz DA, Marks MP, Napel SA, et al. Circle of Willis: evaluation with spiral CT angiography, MR angiography, and conventional angiography. Radiology 1995;195:445-49

16. Waugh JR, Sacharias N. Arteriographic complications in the DSA era. Radiology 1992;182:243-46

17. Prokop M. Multislice CT angiography. Eur J Radiol 2000;36:86-96

18. Karamessini MT, Kagadis GC, Petsas T, et al. CT angiography with three-dimensional techniques for the early diagnosis of intracranial aneurysms: comparison with intra-arterial DSA and the surgical findings. Eur J Radiol 2004;49:212-23

19. Dammert S, Krings T, Moller-Hartmann W, et al. Detection of intracranial aneurysms with multislice CT: comparison with conventional angiography. Neuroradiology 2004;46:427-34

20. Skutta B, Furst G, Eilers J, et al. Intracranial stenoocclusive disease: doubledetector helical CT angiography versus digital subtraction angiography. AJNR Am J Neuroradiol 1999;20:791-95

21. Villablanca JP, Rodriguez FJ, Stockman T, et al. MDCT angiography for detection and quantification of small intracranial arteries: comparison with conventional catheter angiography. AJR Am J Roentgenol 2007;188:593-602

22. Hirai $T$, Korogi $Y$, Ono K, et al. Maximum stenosis of extracranial carotid artery: effect of luminal morphology on stenosis measurement by using CT angiography and conventional DSA. Radiology 2001;221:802-09

23. Ota $\mathrm{H}$, Takase K, Rikimaru $\mathrm{H}$, et al. Quantitative vascular measurements in arterial occlusive disease. Radiographics 2005;25:1141-58

24. Saba L, Caddeo G, Sanfilippo R, et al. Efficacy of axial scans and different reconstruction methods in the study of ulcerated carotid plaque using multidetector-row CT angiography: comparison with surgical results. AJNR Am J Neuroradiol 2007;28:716-23

25. Mallouhi A, Schocke M, Judmaier W, et al. 3D MR angiography of renal arteries: comparison of volume rendering and maximum intensity projection algorithms. Radiology 2002;223:509-16

26. Mallouhi A, Felber S, Chemelli A, et al. Detection and characterization of intracranila aneurysms with MR angiography: comparison of volume rendering and maximum intensity projection. AJR Am J Roentgenol 2003;180:55-64

27. Bae KT, Heiken JP. Scan and contrast administration principles of MDCT. Eur Radiol 2005;15(suppl 5):E46-E59

28. Awai K, Takada K, Onishi H, et al. Aortic and hepatic enhancement and tumor to liver contrast: analysis of the effect of different concentrations of contrast material at multi-detector row helical CT. Radiology 2002;224:757-63 